\title{
Den Flüssen mehr Raum geben - Umsetzungsrestriktionen in Recht und Praxis
}

\author{
Thomas Hartmann
}

Eingegangen: 14. Januar 2011 / Angenommen: 4. April 2011 / Online publiziert: 4. Mai 2011

(C) Die Autor(en) 2011. Dieser Artikel ist auf Springerlink.com mit Open Access verfügbar.

Zusammenfassung In den Jahren 1996, 2005 und zuletzt 2010 traten wichtige Änderungen des Wasserhaushaltsgesetzes in Kraft. Diese hatten jeweils zum Ziel, das wasserwirtschaftliche Planungsinstrumentarium zu stärken, um den Flüssen mehr Raum zu geben. Doch wie wirken diese rechtlichen Änderungen in der Praxis? Der vorliegende Beitrag untersucht dies mit Hilfe einer Politikfeldanalyse am Beispiel der Hochwasserkonzeption des Landes Sachsen-Anhalt. Diese Konzeption wurde erstmals 2003 mit dem Zielhorizont 2010 aufgestellt. Zwei Zeiträume werden betrachtet: vor und nach dem Inkrafttreten des Hochwasserschutzgesetzes 2005. Es wird gezeigt, dass die rechtlichen Änderungen nicht auf die Umsetzungsrestriktionen der Praxis antworten. Die Schwierigkeiten in der Praxis bestehen in erster Linie in den Bereichen, in denen andere Akteure als die Wasserwirtschaft - also etwa die Raumordnung, die Bauleitplanung oder Bodeneigentümer - eine wichtige Rolle bei der Umsetzung der wasserwirtschaftlichen Ziele einnehmen. Der Gesetzgeber folgt bei der Realisierung des räumlichen Hochwasserschutzes (Raum für die Flüsse) einem Kontrollparadigma: Es wird stets versucht, die Instrumente der Wasserwirtschaft zu stärken. Die tatsächlichen Praxisprobleme erfordern jedoch Instrumente, die integrative Planungsprozesse ermöglichen, um Konflikte mit räumlichem Hochwasserschutz zu begegnen und auf ein Umdenken in der Wasserwirtschaft hinzuwirken - von einer kontrollierenden zu einer integrativen Form von Governance. Integrativ bedeutet hier die frühzeitige und aktive Einbindung relevanter Stakeholder in die Planungsprozesse.

Dr. T. Hartmann $(\varangle)$

Faculty of Geosciences, Department of Human Geography

and Planning, Utrecht University, PO-Box 80 115,

3508 TC Utrecht, The Netherlands

E-Mail: t.hartmann@geo.uu.nl
Schlüsselwörter Hochwasserschutz · Wasserwirtschaft · Wasserhaushaltsgesetz $\cdot$ Planungsrecht $\cdot$ Umsetzung

\section{Space for the Rivers-Restrictions for Implementation in Law and Practice}

Abstract In 1996, 2005, and most recently in 2010 the German Federal Water Act was revised. These revisions aimed at stronger instruments to give more space to the rivers. What is their impact in practice? This paper is going to answer this question with the help of a policy analysis along the flood protection concept of the Land SaxonyAnhalt. For the first time, this concept was prepared in 2003, with a planning horizon until 2010. In the analysis, two periods are regarded: before and after the Federal Flood Control Act 2005. It is shown in this article that the law has not responded to the restrictions for implementation in practice. The restrictions, namely, are in a realm where other stakeholders than water management-for example regional planning, local land use planning or landowners - play a key role in the implementation of aims of water management. In the process of spatial flood protection measures, the German legislator pursues a control paradigm: this means that there is a trend to strengthen the instruments of water management in each reform of the law. In fact, however, instruments are needed which would enable integrative planning processes in order to cope with conflicts in spatial flood protection, and in order to support a rethinking in water management-from a technocratic and controlling towards an integrating mode of governance. Integrative means an early and active enrolment of relevant stakeholders in planning processes. 
Keywords Flood protection - Water management · German Federal Water Act · Planning law ·

Implementation

\section{Einführung}

Die Extremhochwasser am Rhein 1993 und 1995 initiierten ein Umdenken im Hochwasserschutz. Die Ministerkonferenz für Raumordnung verwandte1998 in einem Bericht erstmals den Begriff „den Flüssen (mehr) Raum geben“ (MKRO 1998, S. 2)1, den zuvor die niederländische Regierung als Leitbild „Ruimte voor de Rivier“ ihrer Flusspolitik einführte (Greiving 2002, S. 178). Gleichwohl das Leitbild nicht näher konkretisiert und definiert ist, fand der Terminus in den letzten Jahren eine gewisse Popularität in der hochwasserrelevanten Gesetzgebung. So setzte sich die deutsche Bundesregierung mit einem Fünf-Punkte-Programm nach dem Elbehochwasser 2002 das Ziel, den Flüssen mehr Raum zu geben. ${ }^{2}$ Mit dem Gesetz zur Verbesserung des vorbeugenden Hochwasserschutzes (kurz: Hochwasserschutzgesetz) sollte dieses Ziel 2005 umgesetzt werden. Das Gesetz reformierte hauptsächlich Passagen des Wasserrechts, des Bau- und Raumordnungsrechtes. Aber bereits fünf Jahre nach Einführung wurde das Hochwasserschutzgesetz wieder reformiert und teilweise revidiert. Zudem änderte die Föderalismusreform 2006 die Kompetenzverteilung unter anderem im Wasser- und Raumordnungsrecht zwischen Bund und Ländern. „Die sachliche Zusammenarbeit bleibt aufgrund permanenter Umstrukturierungen und Aufgabenverschiebungen auf der Strecke“, erklärte ein Vertreter der Wasserwirtschaft in Sachsen-Anhalt bereits 2004 (Interview Wasserwirtschaft 2004). Nach den Hochwassern 2002 im Einzugsgebiet der Elbe waren auch die Länder an der Umsetzung der Idee interessiert, den Flüssen mehr Raum zu geben. Im Jahr 2003 verabschiedete das Land Sachsen-Anhalt eine Hochwasserschutzkonzeption mit dem Planungsziel 2010. Seit März 2010 ist nun das neue Wasserhaushaltsgesetz in Kraft. Es setzt in erster Linie die Hochwasserrichtlinie $^{3}(2007 / 60 / \mathrm{EG})$ in nationales Recht um. Ein Ziel der Richtlinie ist „den Flüssen mehr Raum zu geben“ (Europäische Union 2007, S. 28). Außerdem verabschiedete das Land Sachsen-Anhalt 2010 die neue Hochwasserschutzkonzeption bis 2020 .

\footnotetext{
${ }^{1}$ Der Grundgedanke, mehr Retentionsraum zu schaffen, war bereits in früheren Entscheidungen der Ministerkonferenz für Raumordnung (MKRO 1995, S. 1; MKRO 1996, S. 1) sowie in Empfehlungen der Länderarbeitsgemeinschaft Wasser (LAWA 1995, S. 7 ff.) enthalten.

${ }^{2}$ Vgl. http://bmu.de/gewaesserschutz/doc/3114.php (letzter Zugriff am 18.12.2010)

${ }^{3}$ Eigentlich: Richtlinie über die Bewertung und das Management von Hochwasserrisiken.
}

Es scheint also angemessen, zu diesem Zeitpunkt die Frage zu stellen, wie das Leitbild „den Flüssen mehr Raum geben" seit dem Hochwasser 2002 verfolgt worden ist. Der vorliegende Beitrag untersucht diese Frage am Beispiel des Landes Sachsen-Anhalt. Hierzu wird zunächst skizziert, wie das Leitbild „den Flüssen mehr Raum geben“ im Laufe der Zeit im Gesetz institutionalisiert wurde, anschließend wird die Umsetzung in der Praxis analysiert. Angelehnt an die Methodik der Politikanalyse nach von Prittwitz (1994) wird basierend auf 2004 und 2008 in Sachsen-Anhalt durchgeführten Experteninterviews hinterfragt, ${ }^{4}$ wie die Gesetzesnovellen die Umsetzung in der Praxis beeinflusst haben. Anschließend wird eine Schlussdiskussion hierzu eröffnet.

\subsection{Umdenken: vom Hochwasserschutz zum Risikomanagement}

Die Idee, den Flüssen mehr Raum zu geben und Wasserwirtschaft ,in der Fläche“ zu betreiben, ist im Laufe der Jahre gewachsen und ist - zumindest formell - nicht älter als rund 15 Jahre. Nach den Hochwasserereignissen in den 1990er Jahren wurde deutlich, dass Deiche alleine nicht vor extremen Hochwassern schützen können und dass mehr Retentionsvolumen nötig ist (Strobl/Zunic 2006, S. 389; Monstadt/Moss 2008, S. 63). Infolgedessen stellte die Länderarbeitsgemeinschaft Wasser (LAWA) 1995 die Leitlinien für zukünftigen Hochwasserschutz auf (LAWA 1995, S. 1). Hochwasserschutz sollte künftig nicht mehr nur technische Maßnahmen wie Deiche, Polder und Rückhaltebecken umfassen, sondern auch räumlichen Hochwasserschutz beinhalten. Hierzu zählen Beschränkungen und Regulierungen der Nutzung von Überschwemmungsgebieten sowie Hochwasservorsorge (Bau- und Flächenvorsorge sowie Verhaltens- und Risikovorsorge) (LAWA 1995). 1998 forderte auch die Ministerkonferenz für Raumordnung (MKRO), künftig regionalplanerische Instrumente für die Bereitstellung von Retentionsräumen entlang der Flüsse zu nutzen und städtebauliche Entwicklungen in hochwassergefährdeten Gebieten zu beschränken (MKRO 1998, S. 1). Die Formel „den Flüssen mehr Raum geben“ wurde zum Leitbild deutscher Flusspolitik. Der Grundgedanke des neuen Leitbildes stellt eine Abkehr von ,keeping the water out" (Johnson/Priest 2008, S. 513) dar und markiert ein Umdenken von „Hochwasserschutz“ zu „Hochwasserrisikomanagement“ (Grünewald 2005). Dieses Umdenken spiegelt sich auch in der hochwasserrelevanten Gesetzgebung - besonders nach dem Extremhochwasser 2002 - wider.

\footnotetext{
${ }^{4}$ Die empirischen Untersuchungen, auf die in diesem Artikel verwiesen wird, wurden im Rahmen der Diplomarbeit sowie der Dissertation des Autors durchgeführt (Hartmann 2005, 2011).
} 


\subsection{Institutionalisierung im Wasserrecht}

Nach den Hochwasserereignissen 2002 beschloss die Bundesregierung erstmals ein umfassendes interministerielles Programm zum Hochwasserschutz: das Fünf-Punkte-Programm (BMVBW 2003, S. 5). Neben der Forderung nach Raum für die Flüsse beinhaltete das Programm Forderungen nach dezentralen Retentionsmaßnahmen und nach einer Reduzierung der Schadenspotenziale durch Beschränkungen in der baulichen Nutzung von Flusslandschaften (Bundesregierung 2005). Außerdem sollen flussgebietsweite Kooperationen auf Ebene der Europäischen Union zu einem besseren Hochwasserschutz beitragen (BMVBW 2003, S. 5). Das Fünf-Punkte-Programm der Bundesregierung kann als nationales Strategiepapier Deutschlands gesehen werden, um den Flüssen mehr Raum zu geben.

Auf dieser Basis wurde 2005 das Gesetz zur Verbesserung des vorbeugenden Hochwasserschutzes erlassen. In der Begründung zum Entwurf des Gesetzes identifizierte die Bundesregierung Regelungs- und Vollzugsdefizite in der Hochwasserflächenvorsorge und im vorbeugenden Hochwasserschutz (BMVBW 2003, S. 13 ff.). Bezüglich des Ziels „den Flüssen mehr Raum geben“ wurde die Sicherung und Wiederherstellung der Flussauen als Ziel der Wasserwirtschaft festgesetzt. Zuvor galt $\S 32$ WHG (Wasserhaushaltsgesetz) in der Fassung von 1996 als wichtigstes wasserwirtschaftliches Instrument zur Sicherung und Wiederherstellung von Überschwemmungsgebieten (Czychowski 1998, S. 1172). Das Instrument war seit 1996 in Kraft, zuvor waren die Sicherung und Wiederherstellung von Überschwemmungsgebieten kein formelles Ziel der Wasserwirtschaft und der Raumplanung (Corell 1996, S. 253). Jedoch hatte die Einführung des § 32 WHG 1996 nicht den erwünschten Effekt. Nur wenige Projekte, den Flüssen mehr Raum zu geben, wurden mit Hilfe dieses Instrumentes realisiert (Umweltbundesamt 2003, S. 150; Breuer 2006, S. 615). Das Hochwasserschutzgesetz sollte 2005 die Möglichkeiten, den Flüssen Raum zu geben, verbessern. Was verursachte die Defizite vor 2005? Wie hat der deutsche Gesetzgeber auf die Probleme 2005 reagiert? Warum wurde das Artikelgesetz zur Verbesserung des vorbeugenden Hochwasserschutzes bereits 2010 durch eine erneute Reform des Wasserhaushaltsgesetzes teilweise revidiert? Im Folgenden werden diese Fragen am Beispiel des Landes Sachsen-Anhalt diskutiert.

\section{Die Hochwasserschutzkonzeption des Landes Sachsen-Anhalt 2003-2010}

Unmittelbar nach dem Hochwasserereignis 2002 verabschiedete das Ministerium für Landwirtschaft und Umwelt des Landes Sachsen-Anhalt ein Hochwasserschutzkonzept, das bis 2010 umgesetzt werden sollte (MLU LSA 2003, S. 3). Die Hochwasserschutzkonzeption beinhaltet die drei Elemente zukunftsweisenden Hochwasserschutzes, wie sie die Landesarbeitsgemeinschaft Wasser 1995 in den Leitlinien benannt hat (LAWA 1995, S. 7 f.): Flächenvorsorge, technischer Hochwasserschutz und Hochwasservorsorge.

In Interviews mit Vertretern der Wasserwirtschaft 2004 wurde deutlich, dass es in der Praxis nach 2002 schwierig war, der Elbe und ihren Nebenflüssen tatsächlich mehr Raum zu geben. Technische Maßnahmen zum Hochwasserschutz hatten Priorität vor Flächenvorsorge. Nach dem Hochwasser waren zwar viele Gelder verfügbar und die politische Unterstützung groß, das Geld musste aber schnell verwendet werden, da die politische Aufmerksamkeit nur von kurzer Dauer war (Interview Wasserwirtschaft 2004). Da aber eine umfassende Flächenvorsorge - nämlich den Flüssen mehr Raum geben - zeit- und kostenaufwendig war und Deichsanierungen mit relativ geringem administrativen Aufwand umgesetzt werden konnten, ${ }^{5}$ wurden nach 2002 die Elbdeiche wieder aufgebaut und verstärkt. Der Sinn einer solchen Vorgehensweise darf hinterfragt werden, insbesondere da ein frisch sanierter und verstärkter Deich schwieriger zu verlegen ist, um den Flüssen mehr Raum zu geben, als ein Deichbauwerk, das womöglich durch ein kürzlich abgelaufenes Hochwasser ohnehin renovierungsbedürftig sein könnte. Nach dem erheblichen Mitteleinsatz und politischen Umsetzungsdruck für besseren Hochwasserschutz 2002 kann daher geschlussfolgert werden, dass die unmittelbaren Maßnahmen nach der Katastrophe die Umsetzung des Leitbildes, den Flüssen mehr Raum zu geben, nachhaltig behindert haben.

Tabelle 1 stellt die Ziele der Hochwasserschutzkonzeption des Landes Sachsen-Anhalt (MLU LSA 2003) und deren Umsetzung vereinfacht dar. Ebenso in der Tabelle dargestellt sind die wasser- und planungsrechtlichen sowie sonstigen Instrumente, die 2004 zur Umsetzung der Konzeption zur Verfügung gestanden haben. Die Regelungen des Landes Sachsen-Anhalt weichen dabei meist nur im Konkretisierungsgrad von der Bundesgesetzgebung ab; so werden im Landeswassergesetz die Zuständigkeiten genauer definiert und detailliertere Bestimmungen zum operativen Hochwasserschutz getroffen (MLU LSA 2010, S. 9). In der Tabelle werden jedoch sowohl die Bundes- als auch landesrechtlichen Bestimmungen aufgeführt. Ferner zeigt die dritte Spalte die tatsächliche Umsetzung der Hochwasserschutzkonzeption. In der letzten Spalte sind Umsetzungsrestriktionen aufgeführt. ${ }^{6}$ Im Folgenden werden die einzelnen Spalten weiter erläutert.

\footnotetext{
${ }^{5}$ Diese fielen nämlich unter $§ 28$ WHG 1996 (Gewässerunterhaltung) und nicht unter § 31 WHG 1996 (Gewässerausbau), was erheblich weniger aufwendig in der administrativen Realisierung war.

${ }^{6}$ Basierend auf Expertengesprächen in 2004.
} 
Tab. 1 Die Umsetzung der Hochwasserschutzkonzeption in Sachsen-Anhalt 2004

\begin{tabular}{|c|c|c|c|c|}
\hline Themen & Ziele & Instrumente & Umsetzung & Restriktionen \\
\hline \multicolumn{5}{|l|}{ Flächenmanagement } \\
\hline \multirow[t]{2}{*}{$\begin{array}{l}\text { Überschwemmungs- } \\
\text { gebiete }\end{array}$} & \multirow[t]{2}{*}{ Gebiete sichern! } & $\begin{array}{l}\text { Wasserwirtschaftliche Festsetzung } \\
(\xi 32 W H G ; \xi 96 W G L S A)\end{array}$ & $+/-$ & $\begin{array}{l}\text { - Häufige Reformen und } \\
\text { Verwaltungsumstrukturierungen } \\
\text { - Interessenskonflikte mit } \\
\text { Bodeneigentümern }\end{array}$ \\
\hline & & $\begin{array}{l}\text { Festsetzung als Vorranggebiete } \\
\text { ( } \$ 7 I V N r .1 \text { ROG; \& } 6 \text { III g LPlG } \\
\text { LSA 1998) }\end{array}$ & $+/-$ & $\begin{array}{l}\text { - Ungeeignete Signaturen (Planzeichen) } \\
\text { - Mangelhafter Datenaustausch }\end{array}$ \\
\hline \multirow[t]{2}{*}{$\begin{array}{l}\text { Deichgeschützte } \\
\text { Gebiete }\end{array}$} & \multirow[t]{2}{*}{$\begin{array}{l}\text { Schadenspotenziale } \\
\text { mindern bzw. nicht } \\
\text { erhöhen! }\end{array}$} & $\begin{array}{l}\text { Vorbehaltsgebiete für Hochwas- } \\
\text { serschutz ( } \$ 7 \text { IV Nr. } 2 \text { ROG; } \S 6 \\
\text { III g LPlG LSA 1998) }\end{array}$ & - & $\begin{array}{l}\text { Konflikte mit kommunaler } \\
\text { Eigenentwicklung }\end{array}$ \\
\hline & & $\begin{array}{l}\text { Bauvorsorge in Bauleitplänen fest- } \\
\text { legen ( } \$ 30 \text { BauGB 2004) }\end{array}$ & - & $\begin{array}{l}\text { - Kommunen erwarten Vorgaben der } \\
\text { Raumordnung } \\
\text { - Vertrauen in Deiche (keine } \\
\text { Notwendigkeit) }\end{array}$ \\
\hline \multirow[t]{3}{*}{$\begin{array}{l}\text { Retention in der } \\
\text { Fläche }\end{array}$} & $\begin{array}{l}\text { Nutzung und Bewirt- } \\
\text { schaftung anpassen! }\end{array}$ & $\begin{array}{l}\text { Hochwasserentstehungsgebiete in } \\
\text { Regionalplänen ausweisen ( } \$ 7 \text { II } \\
\text { ROG 2004; } \$ 6 \text { LPlG LSA 1998) }\end{array}$ & - & $\begin{array}{l}\text { - Wenige Hochwasserentstehungsgebiete } \\
\text { in Sachsen Anhalt } \\
\text { - Konflikte mit der Landwirtschaft }\end{array}$ \\
\hline & $\begin{array}{l}\text { Flächeninanspruchnah- } \\
\text { me begrenzen! }\end{array}$ & $\begin{array}{l}\text { Ausweisung von Siedlungs- } \\
\text { schwerpunkten ( } \$ 7 \text { II ROG 2004; } \\
\text { § } 6 \text { LPlG LSA 1998) }\end{array}$ & $+/-$ & $\begin{array}{l}\text { - Kommunale Eigenentwicklung ist } \\
\text { zugesichert (Art. } 28 \mathrm{GG}) \\
\text { - Politischer Druck durch } \\
\text { kommunalisierte Regionalplanung }\end{array}$ \\
\hline & $\begin{array}{l}\text { Regenwasser vor Ort } \\
\text { versickern! }\end{array}$ & $\begin{array}{l}\text { Festsetzungen in Bebau- } \\
\text { ungsplänen ( } \$ 9 I N r .14 \text { BauGB } \\
\text { 2004) }\end{array}$ & - & $\begin{array}{l}\text { Vorschriften werden von kommunaler } \\
\text { Seite nicht für notwendig erachtet }\end{array}$ \\
\hline \multicolumn{5}{|l|}{ Technischer Schutz } \\
\hline Deichbau & $\begin{array}{l}\text { Deiche erneuern bzw. } \\
\text { sanieren! }\end{array}$ & $\begin{array}{l}\text { Umsetzung als Gewässerunterhal- } \\
\text { tung ( } \$ 28 W H G 1996 ; \S 131 W G \\
L S A \text { 1998) }\end{array}$ & + & \\
\hline $\begin{array}{l}\text { Hochwasserrückhalte- } \\
\text { becken }\end{array}$ & $\begin{array}{l}\text { Hochwasserrückhaltebe- } \\
\text { cken zügig realisieren! }\end{array}$ & $\begin{array}{l}\text { Einleitung der Verfahren ( } \$ 31 \\
W H G \text { 1996; } \$ 131 \text { WG LSA 1998) }\end{array}$ & + & \\
\hline Polder & Flutpolder optimieren! & Verwaltungsinterne Optimierung & + & \\
\hline Unterhaltung & Bauwerke unterhalten! & Bereitstellung finanzieller Mittel & + & \\
\hline \multicolumn{5}{|l|}{ Hochwasservorsorge } \\
\hline Verhaltensvorsorge & $\begin{array}{l}\text { Bauliche Vorsorge } \\
\text { erhöhen! }\end{array}$ & $\begin{array}{l}\text { Informationsmanagement und } \\
\text { partizipative Planung }\end{array}$ & - & $\begin{array}{l}\text { - Vertrauen in technischen Schutz } \\
\text { - Kollektives Vergessen der Gefahr } \\
\text { - Missverständliche Begrifflichkeiten }\end{array}$ \\
\hline \multirow[t]{3}{*}{$\begin{array}{l}\text { Flächen- und } \\
\text { Bauvorsorge }\end{array}$} & $\begin{array}{l}\text { Bauleitpläne an Hoch- } \\
\text { wasserrisiko anpassen! }\end{array}$ & $\begin{array}{l}\text { Integration wasserwirtschaftlicher } \\
\text { Risikoanalysen in kommunale } \\
\text { Planung }\end{array}$ & $+/-$ & $\begin{array}{l}\text { Gefahrenhinweiskarten nicht hinreichend } \\
\text { genau }\end{array}$ \\
\hline & & Restriktive Plangenehmigung & $+/-$ & $\begin{array}{l}\text { Finanzielle Abhängigkeit der Kommunen } \\
\text { von Wachstum }\end{array}$ \\
\hline & $\begin{array}{l}\text { Betroffene sollen bau- } \\
\text { lich vorsorgen! }\end{array}$ & $\begin{array}{l}\text { Bauvorsorge in Bauleitplänen fest- } \\
\text { legen }(\S 30 \text { BauGB 2004) }\end{array}$ & - & $\begin{array}{l}\text { Kommunen sehen Eigenverantwortlich- } \\
\text { keit der Betroffenen }\end{array}$ \\
\hline Risikovorsorge & $\begin{array}{l}\text { Versichertenquote } \\
\text { erhöhen! }\end{array}$ & Keine Instrumente & - & Kein Akteur fühlt sich zuständig \\
\hline
\end{tabular}

\subsection{Ziele der Hochwasserschutzkonzeption 2003}

Die Hochwasserschutzkonzeption des Landes SachsenAnhalt hatte sich zum Ziel gesetzt, den „natürlichen Hochwasserrückhalt auf der Fläche“ zu verbessern und insofern den Flüssen mehr Raum zu geben (MLU LSA 2003, S. 4). In Bezug auf räumliche Maßnahmen zum Hochwasserschutz wurden daher Ziele zur Festsetzung von Überschwemmungsgebieten, zur Reduzierung von Schadenspotenzialen auch in deichgeschützten Gebieten, zur Anpassung der Bodennutzung in der Fläche, zur Reduzierung des Flächenverbrauchs und zu dezentralen Rückhaltemaßnahmen aufgestellt (vgl. Tab. 1). Das Land hatte sich 2003 vorgenommen, die Ziele bis 2010 umzusetzen. Außerdem sollten Maßnahmen zur Hochwasservorsorge realisiert werden: Die Hochwasserwahrnehmung sollte erhöht werden, die Flächen- und Bauvorsorge sollten verbessert werden und schließlich setzte sich das Land das (nicht näher quantifizierte) Ziel, die Versi- 
chertenquote in Sachsen-Anhalt zu erhöhen, um Risikovorsorge zu betreiben (MLU LSA 2003, S. 64). Der technische Hochwasserschutz spielte 2003 eine zentrale Rolle. Im Rückblick stellt die aktuelle Hochwasserschutzkonzeption aus 2010 (bis 2020) fest, dass die Hochwasserschutzkonzeption 2003 „mit Aussagen zu Schadensbeseitigung und deutlichem Nachholbedarf im Hochwasserschutz geprägt“ war (MLU LSA 2010, S. 21).

\subsection{Umsetzungsinstrumente in 2004}

Welche Instrumente standen im Jahr 2004 zur Umsetzung der Hochwasserschutzkonzeption zur Verfügung? Ziele des technischen Hochwasserschutzes beinhalten Maßnahmen im originären Handlungsfeld der Wasserwirtschaft (Monstadt/Moss 2008, S. 70), während die Ziele aus den Bereichen Hochwasserflächenmanagement und Hochwasservorsorge - die für das Leitbild „,den Flüssen mehr Raum geben" essentiell sind - Kooperation mit Raumordnung, kommunaler Bauleitplanung oder Bodeneigentümern erfordert. In Tab. 1 sind den Zielen der Hochwasserschutzkonzeption aus den Bereichen Hochwasserflächenmanagement, technischer Hochwasserschutz und Hochwasservorsorge Umsetzungsinstrumente zugeordnet.

Das Umweltbundesamt stellte 1999 bereits fest, dass die Instrumente des Wasserhaushaltsgesetzes 1996 prinzipiell ausreichend waren, um den Flüssen mehr Raum zu geben (Umweltbundesamt 1999, S. VIII), wobei jedoch einige Instrumente zur Umsetzung der Hochwasserschutzkonzeption nicht originär aus dem Wasserrecht stammen, sondern auch aus dem Baugesetzbuch oder dem Raumordnungsgesetz (Haupter/Heiland 2002, S. 77; Schneider 2005, S. 5 f.). Um also den Flüssen in Deutschland mehr Raum zu geben, war es auch 2004 erforderlich, mit unterschiedlichen raumrelevanten Akteuren zu kooperieren und diese zu koordinieren.

\subsection{Handlungsbilanz 2004}

2004 waren die Ziele aus dem Bereich des technischen Hochwasserschutzes entweder bereits umgesetzt oder sehr weit fortgeschritten, während bei den Zielen in den beiden anderen Handlungsfeldern deutliche Umsetzungsdefizite zu verzeichnen waren. Tabelle 1 zeigt den Stand der Umsetzung der einzelnen Ziele in 2004. Ein „+" zeigt an, dass die Umsetzung der Ziele der Hochwasserschutzkonzeption weit fortgeschritten ist, ,+/-“ bedeutet eine mäßige Zielerreichung, ,-“ steht für eine zögerliche oder langwierige Zielumsetzung. Im Allgemeinen werden Ziele außerhalb der Umsetzungskompetenz der Wasserwirtschaft nicht oder nur rudimentär erreicht. Es bestanden also Defizite bei der Koordination und im Vollzug von Regelungen zum vorsor- genden Hochwasserschutz (vgl. Umweltbundesamt 1999, S. VIII).

So ist zum Beispiel die wasserwirtschaftliche Festsetzung von Überschwemmungsgebieten nach § 96 WG LSA (Wassergesetz für das Land Sachsen-Anhalt) 1998 zu nennen. Zwar waren im November 2004 alle Überschwemmungsgebiete durch § 96 IIIa WG LSA 1998 faktisch gesichert (per Definition im Gesetzestext), allerdings existierte nur für einen Teil dieser Gebiete eine Verordnung, wie es § 96 I WG LSA 1998 vorschreibt (Interview Wasserwirtschaft 2004). Begründet mit der langen Verfahrensdauer solcher wasserwirtschaftlicher Festsetzungen von Überschwemmungsgebieten (Landtag Sachsen-Anhalt 2003, S. 40), wurde als Reaktion auf das Hochwasser 2002 im Rahmen des Zweiten Investitionserleichterungsgesetzes die vorläufige Sicherung von faktischen Überschwemmungsgebieten bis 2010 festgelegt, um die Sicherung von Überschwemmungsgebieten per Verordnung zu ermöglichen (GVB1. LSA 2003, S. 164). Die in der Definition enthaltenen Gebiete beziehen sich jedoch ausschließlich auf bestehende Überschwemmungsgebiete - diese Regelungen waren also nicht dazu bestimmt, den Flüssen tatsächlich mehr Raum zu geben.

Bei Festlegungen zur Umsetzung der Ziele der Hochwasserschutzkonzeption in Bauleit- und Regionalplänen zeigte sich im Jahr 2004 eine nur sehr zögerliche und im Land uneinheitliche Anwendung des möglichen Instrumentariums. § 96 II WG LSA 1998 in Verbindung mit § 48 Abs. 6 WG LSA 1998 verlangte zwar, Überschwemmungsgebiete in die Raumordnungsplanung aufzunehmen, doch war die Form der Aufnahme nicht näher bestimmt. Auch wurden in Interviews mit wasserwirtschaftlichen Vertretern 2004 der zu grobe Maßstab und die flurstücksunscharfe Festlegung im Landesentwicklungsplan bemängelt (Interview Wasserwirtschaft 2004). Auf der Ebene der Regionalplanung wurde 2004 das Instrument der Vorbehaltsgebiete zur vorläufigen Sicherung von Überschwemmungsgebieten kaum genutzt, da es als zu schwach eingestuft wurde (Interviews Regionalplanung 2004). Dies wird auch für andere Bundesländer bestätigt: Obwohl „das Bewusstsein für die Notwendigkeit des Belanges Hochwasserschutz in der Raumordnung generell bei Planungsverantwortlichen vorhanden ist" (Heiland 2002, S. 125), wurde in zahlreichen Raumordnungsplänen das vorhandene Instrumentarium nur in geringem Umfang verwendet.

Es lässt sich zusammenfassen, dass 2004 diejenigen Ziele der Hochwasserschutzkonzeption, die den Flüssen mehr Raum geben sollten, nicht erreicht wurden, wohingegen technischer Hochwasserschutz sehr weit fortgeschritten war. Auch das Land Sachsen-Anhalt bestätigt in seiner Hochwasserschutzkonzeption 2010-2020 die Einschätzung, dass die Hochwasserschutzkonzeption aus 2003 einen Schwerpunkt im technischen Hochwasserschutz hatte: „In 
den Jahren 2002-2006 dominierte die Hochwasserschadensbeseitigung" (MLU LSA 2010, S. 5)

\subsection{Restriktionen der Umsetzung 2004}

Die Trennung in räumliche Gesamtplanung und Fachplanungen erforderte einen hohen Koordinationsaufwand (Greiving 2002, S. 136). Das Umweltbundesamt sah ein Problem der Umsetzung auf der Ebene der lokalen Planung: Die „,insgesamt heterogene und unsichere Handhabung vieler Städte, Gemeinden und Kreise bei der Umsetzung veränderter gesetzlicher Anforderungen und Aufgabenzuweisungen sowie überörtlicher Planungsvorgaben ist möglicherweise ein Indikator dafür, dass die ,Botschaften von oben nach unten noch nicht richtig angekommen sind“" (Umweltbundesamt 2003, S. 148). Unterschiede in der individuellen Bedeutungsgebung des Hochwasserschutzes gegenüber anderen Belangen (vgl. Umweltbundesamt 1998, S. 201) sowie unterschiedliche Zuständigkeiten und Landesgesetze schränkten eine effektive Zusammenarbeit entlang eines Flusses ein. Instrumente zur grenzüberschreitenden Aufstellung und Umsetzung von Planungszielen wurden als nicht ausreichend bemängelt (Umweltbundesamt 1999, S. 36 f.).

Auch die im Rahmen der Studie befragten Akteure aus Wasserwirtschaft, Regional- und Landesplanung sowie kommunaler Planung in Sachsen-Anhalt erklärten 2004, dass die Instrumente, um den Flüssen mehr Raum zu geben, zwar prinzipiell ausreichend gewesen seinen, jedoch eine intensive Zusammenarbeit aller Akteure erforderten. Regionalplaner führten an, dass notwendige Informationen seitens der Fachplanung nicht oder in unbrauchbaren Formaten zur Verfügung gestellt worden seien. Ohne solche wasserwirtschaftlichen Informationen seien raumordnerische Ausweisungen und Beschränkungen, um den Flüssen Raum zu geben, nicht realisierbar. Planer auf kommunaler Ebene wiederum erwarteten Vorgaben seitens der Raumordnung, um entsprechend restriktive Bauleitpläne aufzustellen bzw. eine weitere Besiedlung der Flusslandschaften zu reduzieren. Die wasserwirtschaftliche Festsetzung von Überschwemmungsgebieten nach $\S 32$ WHG 1996 sei zu restriktiv gegenüber Bodeneigentümern und daher sehr langwierig. Die Wasserwirtschaft wiederum beklagte das Dilemma zwischen Deregulierung und notwendigen wasserwirtschaftlichen Beschränkungen. Alle befragten Akteure beklagten den Mangel an Koordination und Kooperation und wünschten sich untereinander mehr davon.

Es wurde in der empirischen Untersuchung 2004 auch deutlich, dass unterschiedliche Problemauffassungen und ein unterschiedliches Planungsverständnis der Akteure die Zusammenarbeit erschwerten. Stellung in der Verwaltung, Ausbildung, Erfahrung und individuelle Problemwahrnehmung begründeten unterschiedliche Handlungsrationalitäten der Akteure. Diese Rationalitäten spielten eine bedeutende
Rolle bei der Umsetzung komplexer Planungsziele (vgl. dazu näher Hartmann 2010, 2011).

\section{Neue Gesetzgebung 2005}

2005 reagierte der deutsche Gesetzgeber auf die Probleme in der Umsetzung durch das Artikelgesetz zur Verbesserung des vorbeugenden Hochwasserschutzes (Hochwasserschutzgesetz). In erster Linie reformierte und konkretisierte das Hochwasserschutzgesetz hochwasserrelevante Passagen im Wasserhaushaltsgesetz, im Baugesetzbuch und im Raumordnungsgesetz. Das Wassergesetz des Landes Sachsen-Anhalt wurde mit der Bekanntmachung vom 22. April 2006 mit Wirkung zum 12. April 2005 entsprechend angepasst (GVBl. LSA 2006, S. 248). Der Landesgesetzgeber reagierte damit ebenso auf die Hochwasserereignisse 2002. 2008 waren allerdings die bundesrechtlichen Regelungen des Wasserhaushaltsgesetzes durch die Föderalismusreform 2006 von vorrangiger Bedeutung für das Land SachsenAnhalt, da die Reform durch die Aufhebung der Rahmengesetzgebungskompetenz des Bundes im Bereich Wasser das Wasserhaushaltsgesetz zur Vollregelung erhob (Pape 2010, Rn 64). Tabelle $2^{7}$ zeigt, welche Regelungen des reformierten Rechts die Umsetzungsoptionen der Hochwasserschutzkonzeption des Landes Sachsen-Anhalt veränderten.

\subsection{Umsetzungsinstrumente ab 2005}

Das Hochwasserschutzgesetz 2005 führte eine neue Gebietskategorie ins Wasserrecht ein: überschwemmungsgefährdete Gebiete (§31c II WHG 2005). Diese Raumkategorie war erstmals ein Versuch, hochwasserrelevante Festlegungen der Wasserwirtschaft zur Raumplanung hinter den Deichen im Gesetz zu verankern. ${ }^{8}$ Zusätzlich verlangte das Hochwasserschutzgesetz von der Wasserwirtschaft die Erstellung von Hochwasserschutzplänen bis 2010 bzw. 2012 (§ 31d WHG 2005). Auch das Bauen in flussnahen Bereichen unterlag strengeren Regelungen. Bodeneigentümer wurden nun auch im Gesetz in die Verantwortung zur Mitwirkung gezogen. Raumplaner sollten für das Thema Hochwasserrisiko sensibilisiert werden; wasserwirtschaftliche Planungen sollten stärker in die Raumplanung integriert werden. Letztendlich stärkte die Gesetzgebung 2005 die formellen Instrumente des Hochwasserschutzes (Cormann 2008, § 31a WHG Rn 2). Dennoch, auch das Artikelgesetz zur Verbesserung des vorbeugenden Hochwasserschutzes vermochte kein generelles Bauverbot für hochwassergefährdete Gebiete zu formulieren.

\footnotetext{
${ }^{7}$ Technischer Hochwasserschutz wies bereits 2004 einen guten Umsetzungsstand auf und wird daher in der Tabelle nicht berücksichtigt.

${ }^{8}$ Bisherige Festlegungen gingen meist erst durch raumordnerische Abwägung, z. B. als Vorbehaltsgebiete in die Raumplanung ein.
} 
Tab. 2 Die Umsetzung der Hochwasserschutzkonzeption in Sachsen-Anhalt 2004

\begin{tabular}{|c|c|c|c|c|}
\hline Themen & Ziele & Instrumente & Umsetzung & Restriktionen \\
\hline \multicolumn{5}{|l|}{ Flächenmanagement } \\
\hline \multirow[t]{3}{*}{$\begin{array}{l}\text { Überschwemmungs- } \\
\text { gebiete }\end{array}$} & \multirow[t]{3}{*}{ Gebiete sichern! } & $\begin{array}{l}\text { Frist zur Ausweisung ( } \$ 31 b W H G \\
\text { 2005; } \$ 96 \text { VI WG LSA 2006) }\end{array}$ & $++/-$ & $\begin{array}{l}\text { Keine Konsequenzen bei } \\
\text { Nichterfüllung }\end{array}$ \\
\hline & & $\begin{array}{l}\text { Strengere Regulierungen zum Bauen } \\
\text { ( } \$ 31 b W H G 2005 ; \S 96 W G L S A \\
2006)\end{array}$ & & $\begin{array}{l}\text { Konflikte durch strengere } \\
\text { Regulierungen verschärft }\end{array}$ \\
\hline & & $\begin{array}{l}\text { Gebot zur Übernahme der Hochwasser- } \\
\text { belange in Raumordnungsplänen ( } \$ 7 \\
\text { III ROG 2005) }\end{array}$ & $+/-$ & $\begin{array}{l}\text { Interessenskonflikte, dadurch } \\
\text { langwierige Verfahren }\end{array}$ \\
\hline $\begin{array}{l}\text { Deichgeschützte } \\
\text { Gebiete }\end{array}$ & $\begin{array}{l}\text { Schadenspotenziale } \\
\text { mindern bzw. nicht } \\
\text { erhöhen! }\end{array}$ & $\begin{array}{l}\text { Neue Kategorie: überschwemmungs- } \\
\text { gefährdete Gebiete ( } \$ 31 c \text { WHG } 2005 \text {; } \\
\S 98 \text { a WG LSA 2006) }\end{array}$ & - & $\begin{array}{l}\text { - Schwaches Instrument } \\
\text { - Keine Wirkung auf bereits } \\
\text { bebaute Gebiete }\end{array}$ \\
\hline \multirow[t]{3}{*}{$\begin{array}{l}\text { Retention in der } \\
\text { Fläche }\end{array}$} & $\begin{array}{l}\text { Nutzung und Bewirt- } \\
\text { schaftung anpassen! }\end{array}$ & $\begin{array}{l}\text { Bewirtschaftungsgebote sind im Ge- } \\
\text { setzgebungsverfahren gekippt }\end{array}$ & - & Keine Instrumente verfügbar \\
\hline & $\begin{array}{l}\text { Flächeninanspruchnah- } \\
\text { me begrenzen! }\end{array}$ & Keine neuen Instrumente & $+/-$ & Keine Änderung \\
\hline & $\begin{array}{l}\text { Regenwasser vor Ort } \\
\text { versickern! }\end{array}$ & $\begin{array}{l}\text { Hochwasserbelange müssen in Bebau- } \\
\text { ungsplänen berücksichtigt werden }(\xi 1 \\
V I N r .12 \text { BauGB 2005) }\end{array}$ & $+/-$ & $\begin{array}{l}\text { - Schwaches Instrument } \\
\text { - Vertrauen in Deiche (keine } \\
\text { Notwendigkeit) }\end{array}$ \\
\hline \multicolumn{5}{|l|}{ Hochwasservorsorge } \\
\hline Verhaltensvorsorge & $\begin{array}{l}\text { Risikobewusstsein der } \\
\text { Betroffenen stärken }\end{array}$ & $\begin{array}{l}\text { Keine neuen Instrumente (ggf. } \S 31 a \text { II } \\
\left.W H G 2005^{*}\right)\end{array}$ & - & Keine Änderung \\
\hline \multirow[t]{2}{*}{$\begin{array}{l}\text { Flächen- und } \\
\text { Bauvorsorge }\end{array}$} & $\begin{array}{l}\text { Bauleitpläne an Hoch- } \\
\text { wasserrisiko anpassen! }\end{array}$ & $\begin{array}{l}\text { Hochwassergefahr muss in Plänen } \\
\text { dargestellt werden ( } \$ 5 \text { und } \$ 9 \text { BauGB } \\
\text { 2005; } \$ 7 \text { ROG 2005) }\end{array}$ & $+/-$ & $\begin{array}{l}\text { Darstellungen führen nicht zu } \\
\text { Restriktionen beim Bauen }\end{array}$ \\
\hline & $\begin{array}{l}\text { Betroffene sollen bau- } \\
\text { lich vorsorgen! }\end{array}$ & Keine neuen Instrumente & - & Keine Änderung \\
\hline Risikovorsorge & $\begin{array}{l}\text { Versichertenquote } \\
\text { erhöhen! }\end{array}$ & Keine neuen Instrumente & - & Keine Änderung \\
\hline
\end{tabular}

a Zwar zielt §31a Abs. 2 WHG 2005 in Richtung einer Verhaltensvorsorge von Betroffenen, da die Bestimmung Jedermann verpflichtet, geeignete Vorsorgemaßnahmen zu treffen, jedoch wurden an die Missachtung dieser Verpflichtung keinerlei unmittelbare Rechtsfolgen für Einzelne geknüpft. Daher muss dieser Absatz eher als Haftungsausschluss des Staates für individuelle Hochwasserschäden interpretiert werden.

Schlussendlich führte die neue Gesetzgebung zwar stärkere Instrumente für die Wasserwirtschaft ein - so wurden beispielsweise administrative Hürden bei Deichrückverlegungen abgebaut (MLU LSA 2010, S. 32). Die raumplanerischen Instrumente wurden jedoch kaum im Sinne einer Politik, die den Flüssen Raum geben möchte, gestärkt. Tabelle 2 ordnet die Instrumente den Zielen der Hochwasserschutzkonzeption Sachsen-Anhalts zu.

\subsection{Handlungsbilanz 2008}

2008 wurden die gleichen Akteure wie 2004 ein zweites Mal zur Umsetzung von „Raum für die Flüsse“ in Sachsen-Anhalt befragt. Was hatte sich seit 2004 getan? 2008 sollte die Hochwasserschutzkonzeption des Landes Sachsen-Anhalt nahezu umgesetzt sein - der Zielhorizont war 2010. Aber die Maßnahmen, um den Flüssen mehr Raum zu geben, wurden immer noch zögerlich realisiert - jedoch nicht nur in Sachsen-Anhalt, in ganz Deutschland ging und geht die Umsetzung nur langsam vonstatten (Monstadt/ Moss 2008, S. 64). Trotz der Intention des Gesetzgebers, mit dem Hochwasserschutzgesetz Regelungen einzuführen, um den Flüssen mehr Raum zu geben, zeigte die zweite Befragung von Experten und Bodeneigentümern in Sachsen-Anhalt in 2008, dass es offensichtlich immer noch schwierig war, wirksam raumplanerisch zu intervenieren. Dies belegt die Aussage eines Regionalplaners anschaulich: „Die Regionalplanung muss sehr kämpfen, um Bautätigkeit in überschwemmungsgefährdeten Gebieten zu verhindern" (Interviews Regionalplanung 2008). Bürgermeister besonders von kleineren Kommunen waren - entgegen der Position des Bundesgesetzgebers - auch 2008 davon überzeugt, dass Flusslandschaften als günstiges und attraktives Bauland genutzt werden sollten. Regionalplanerische oder wasserwirtschaftliche Beschränkungen dieser Bautätigkeit wurden als Hindernis kommunaler Eigenentwicklung angesehen. Das Hochwasserschutzgesetz 2005 hatte also nicht den erhofften Effekt der Sensibilisierung von Planern; Flusslandschaften galten nach wie vor als attraktive Flächen für städtebauliche Entwicklungen (Petrow/Thieke/Kreibich et al. 2006, S. 717). 


\subsection{Restriktionen der Umsetzung 2008}

Worin liegen die Gründe hierfür? Das Artikelgesetz hatte zum Ziel, ein besseres wasserwirtschaftliches und raumplanerisches Instrumentarium zu entwickeln, um den Flüssen Raum zu geben. Offensichtlich wurde das zur Verfügung stehende Instrumentarium - ebenso wie vor der Reform nur zögerlich angewandt, denn das Hochwasserschutzgesetz führte nicht zu essentiell restriktiverer Ausweisungspolitik in Überschwemmungsgebieten und überschwemmungsgefährdeten Gebieten. Auch flächendeckende bauliche Vorsorgemaßnahmen waren nicht zu beobachten. So zeigten zum Beispiel im Rahmen der empirischen Erhebung befragte Bodeneigentümer teilweise sehr stolz, wie durch das Hochwasser zerstörte Parkettböden wieder hergestellt worden waren - nicht an die Überschwemmungsgefahr angepasst, finanziert durch Spendengelder. ${ }^{9}$ Zwar entstanden seit 2005 auch weitere technische Maßnahmen zum Hochwasserschutz, doch hier hatten ohnehin keine so großen Umsetzungsrestriktionen bestanden.

Seitens der Wasserwirtschaft erklärten die befragten Akteure, dass die Instrumente zu schwach seien, um attraktive Baugebiete in überschwemmungsgefährdeten Gebieten zu verhindern. Auch in der Begründung zum Wassergesetz des Landes Sachsen-Anhalt wird erklärt, dass diese Gebietskategorie - die bereits 2004 in das Landeswassergesetz eingeführt wurde - lediglich hinweisenden Charakter für die räumliche Planung hat (Landtag Sachsen-Anhalt 2004, S. 75). Darüber hinaus beklagten Vertreter der Wasserwirtschaft, es gäbe keine Instrumente, um in bereits bebauten Gebieten wirksame Hochwasservorsorge umzusetzen. Der Widerstand der Bodeneigentümer sei die schwierigste Restriktion, um den Flüssen mehr Raum zu geben, so erklärten Regionalplanung und Wasserwirtschaftsverwaltung in den Interviews 2008 (Interviews Regionalplanung 2008; Interviews Wasserwirtschaft 2008). Die Nachfrage nach Bauland am Wasser sei noch ungebrochen (Interviews Stadtplanung 2008). Jedoch wurde auch deutlich, dass Wasserwirtschaft, Raumordnung und Kommunalplanung unterschiedliche Problemwahrnehmungen hatten. Karten, die auf die Überschwemmungsgefahr oder wasserwirtschaftliche Beschränkungen in deichgeschützten Gebieten hinwiesen, wurden von vielen Kommunen als Gefahr für die wirtschaftliche Entwicklung von Städten und Gemeinden angesehen (Interviews Stadtplanung 2008). Insgesamt zeigte sich in den Interviews, dass das Vertrauen in Deiche bei Stadtplanern ungebrochen ist: „Alles was im deichgeschützten Bereich liegt, ist Siedlungsgebiet. Da gibt es keinen Grund, keine Bauflächen auszuweisen“ (Interview Stadtplanung 2008). Auf Ebene der Landesentwicklung Sachsen-Anhalt wurde betont, dass Hochwasserschutz ein wichtiges Thema der Lan-

\footnotetext{
${ }_{9}^{9}$ Das Beispiel stammt aus Dessau-Waldersee.
}

desentwicklung sei, „es ist aber nicht wichtiger als andere“ (Interview Landesplanung 2008). Die Wasserwirtschaft beklagte auch 2008, dass Beschränkungen von Siedlungsaktivitäten oder verbindliche Regelungen zu angepasstem Bauen hinter den Deichen schwierig seien. „Warum diese Bautätigkeit nicht zu stoppen ist, ist schwierig zu beantworten“ (Interview Wasserwirtschaft 2008).

\section{Resümee: 15 Jahre Raum für die Flüsse}

„Der Hochwasserschutz ist ins Wasser gefallen - an der Elbe sind fünf Jahre nach der Jahrhundertflut Deiche erhöht - Forderungen nach mehr Raum für den Fluß bleiben unerfüllt“ - so betitelte die regionale Tageszeitung „Die Rheinpfalz“ am 29. Juni 2007 einen Artikel über den Hochwasserschutz in Deutschland. Die empirischen Untersuchungen in Sachsen-Anhalt bestätigen diese Einschätzung. Trotz der Zielsetzungen des Landes nach 2002, Flächen für den Hochwasserrückhalt bereitzustellen und Vorsorgemaßnahmen für deichgeschützte Flächen festzulegen (MLU LSA 2003, S. 35 f.), ist festzustellen, dass nach Ablauf der selbst gesetzten Frist bis 2010 raumplanerische Beschränkungen in der Fläche nur sehr zögerlich angewendet wurden, während die Realisierung von wasserwirtschaftlichen Bauten wie der Bau und die Unterhaltung von Deichen verhältnismäßig reibungslos funktionierte.

Schlussendlich scheint deutsches Flussgebietsmanagement über 15 Jahre nach Einführung der Idee „Raum für die Flüsse" in die politische Diskussion immer noch deichfixiert zu sein. Auch das Hochwasserschutzgesetz 2005 vermochte nicht, den Flüssen tatsächlich mehr Raum zu geben. Vielmehr verschärften die strengeren Regelungen zum Bauen in Überschwemmungsgebieten sowie die Angst vor Restriktionen durch die Ausweisung von überschwemmungsgefährdeten Gebieten die Konflikte zwischen Hochwasserschutz und wirtschaftlicher Nutzung des Bodens. Das Artikelgesetz führte also letztendlich nicht zu einer Erleichterung bei der Umsetzung - die 2004 beschriebenen Restriktionen für die Akteure hätten Deregulierung, Instrumente zur Konfliktlösung wirtschaftlicher Interessensdifferenzen und auch eine gewisse Gelassenheit erfordert. Stattdessen führte das Hochwasserschutzgesetz noch restriktivere Instrumente ein. Der Trend zur stetigen und einseitigen ${ }^{10}$ Verschärfung der gesetzlichen Regelungen ${ }^{11}$ zum Hochwasserschutz und der damit einhergehende Versuch, die Flussräume durch wasserwirtschaftsplanerische Interventionen zu kontrollieren, kann als eine „,command and control“-

\footnotetext{
${ }^{10}$ Nämlich auf Seiten der Wasserwirtschaft.

${ }^{11}$ Wie eindrücklich an der Entstehungsgeschichte der ursprünglichen Bestimmung § 32 WHG 1996 (Überschwemmungsgebiete) bis hin zu $\S 76$ WHG 2010 nachvollzogen werden kann.
} 
Rationalität bezeichnet werden (Roth/Warner 2007, S. 519), die die deutsche Hochwassergesetzgebung dominiert (Jacobitz 2005, S. 1088). Dabei zielt die Rhetorik in der politischen Hochwasserschutzdebatte eher auf kooperative und koordinierende Instrumente ab. Zum Beispiel werden im Projekt ELLA (Elbe/Labe), in dem auch zahlreiche Ministerien und öffentliche Institutionen Partner sind, alle Akteure aufgefordert „in Kooperationen und gegebenenfalls sogar in transnationale Partnerschaften einzutreten“" (Sächsisches Staatsministerium des Innern 2006, S. 4 f.).

Wenn nun also schon das Gesetz nicht auf die konkreten Umsetzungsrestriktionen der Praxis Rücksicht zu nehmen scheint, so darf die Frage gestellt werden, mit welchen Bestimmungen dem Ziel, den Flüssen mehr Raum zu geben, nun brauchbare Instrumente geschaffen wurden.

\subsection{Das Wasserhaushaltsgesetz 2010 - ein neuer Anlauf}

Im März 2010 ist das neue Wasserhaushaltsgesetz in Kraft getreten. Es soll das Wasserrecht reformieren und stärken (Deutscher Bundestag 2009a, S. 1). Das Wasserrecht wurde bereits lange Zeit als zu fragmentiert (Schneider 2005, S. 4), dezentralisiert und komplex kritisiert (Monstadt/Moss 2008, S. 64 f.). Die deutsche Bundesregierung präsentiert das neue Gesetz systematisierter und einheitlicher (Deutscher Bundestag 2009a, S. 1) als die bisherigen Regelungen. ${ }^{12}$

Der erst 2005 veränderte und teilweise neu eingeführte Abschnitt über Hochwasserschutz wurde nun grundlegend reformiert. Erstmalig definiert der Gesetzgeber nun die Begriffe „Hochwasser“ (§ 72 WHG 2010) und „Risiko“ (§ 73 I WHG 2010) entsprechend der Europäischen Hochwasserrisikomanagementrichtlinie 2007/60/EG. So führt das neue Gesetz auch das Instrument der Hochwasserrisikomanagementpläne ein, die bis 2015 aufgestellt werden sollen (§ 75 WHG 2010). Diese Pläne sollen Ziele und Maßnahmen zur Reduzierung von Hochwassergefahren beinhalten und lösen die 2005 neu eingeführten „Hochwasserschutzpläne“ ab. Bezogen auf das Ziel, den Flüssen mehr Raum zu geben, war die alte Regelung jedoch deutlicher: Hochwasserschutzpläne zielten ,insbesondere [auf die] Rückgewinnung von Rückhalteflächen“ (§ 31d WHG 2005) - eine Formulierung, die im Gesetzestext zu Risikomanagementplänen nicht enthalten ist. Zwar wurde eine solche Formulierung durchaus im Gesetzgebungsverfahren diskutiert (vgl. Deutscher Bundestag 2009b, S. 3), aber schlussendlich nicht eingeführt. ${ }^{13} \mathrm{Ob}$ die Erforderlichkeitsbestimmung nach $\S 75$ II WHG 2010, wonach „nichtbauliche Maßnahmen“ vorzuse-

\footnotetext{
${ }^{12}$ Auf den langen und schwierigen Entstehungsweg des Gesetzes als Ergebnis des Scheiterns des Umweltgesetzbuches soll hier nicht näher eingegangen werden; vgl. dazu ausführlich Knopp (2010).

${ }^{13}$ Wobei die Richtlinie der Europäischen Union den Begriff „,den Flüssen Raum geben“ in der Richtlinienbegründung durchaus explizit aufgreift (Europäische Union 2007, S. 28).
}

hen sind, sofern es erforderlich ist, ein sinnvolles Substitut darstellt, darf bezweifelt werden. So ist beispielsweise die Hochwasserschutzkonzeption des Landes Sachsen-Anhalt bis 2020 weiterhin sehr vorsichtig mit konkreten Zielen, um den Flüssen Raum zu geben. Die Formulierungen zur Flächenvorsorge verbleiben im Rahmen von „kann“-Aussagen und auch die raumordnerische Ausweisung von Flächen, um den Flüssen mehr Raum zu geben, wird gegenüber technischen Zielen in der Konzeption nur zurückhaltend und vage formuliert (MLU LSA 2010, S. 66 ff. und 74), Maßnahmen zur Bauvorsorge werden lediglich empfohlen und auch zur Risikovorsorge finden sich in der Konzeption lediglich Verweise auf eine Zusammenarbeit mit der Versicherungswirtschaft bei der Datenbeschaffung und -aktualisierung (MLU LSA 2010, S. 90 und 99).

Es zeigt sich also, dass auch 2010 die Aussagen im Kompetenzbereich der Wasserwirtschaft sehr detailliert und konkret sind, während Ziele, die eine Realisierung durch andere erfordern, eher unkonkret bleiben. Es scheint, dass die Wasserwirtschaftsverwaltungen noch nicht auf eine integrative Planungsweise, die auch Stakeholder aus anderen Fachpolitiken einbezieht, eingestellt sind.

Nach wie vor das wichtigste Instrument scheint die Festsetzung von Überschwemmungsgebieten zu sein (ehemals $\S 32$ WHG 1996 dann § 31b WHG 2005, nun § 76 WHG 2010). Allerdings ist dieses Instrument nur sehr eingeschränkt in der Lage, den Flüssen mehr Raum zu geben, da deichgeschützte Bereiche nicht als Überschwemmungsgebiet festgesetzt werden können, wie bereits die Ministerkonferenz für Raumordnung im Jahre 2000 festgestellt hat (MKRO 2000, S. 10). Zudem ist die wasserwirtschaftliche Festsetzung als Überschwemmungsgebiet aufgrund der starken Widerstände von Kommunen und Bodeneigentümern sehr langwierig. So waren in 2010 erst 14,6\% der Überschwemmungsgebiete in Sachsen-Anhalt per Verordnung festgestellt (MLU LSA 2010, S. 66) - 2003 waren es 12,6\% (MLU LSA 2003, S. 35). Das Hochwasserschutzgesetz versuchte, dem durch Festsetzung einer Umsetzungsfrist - ganz im Sinne des oben erwähnten ,command and control"-Ansatzes - Herr zu werden. Da jedoch keine Konsequenzen für die Nichterreichung dieser Frist festgesetzt wurden, stand diese Regelung seit Beginn in der Kritik (Kotulla 2006, S. 130). Diese Kritik wird durch die Tatsache untermauert, dass die Frist bereits $2009^{14}$ außer Kraft gesetzt wurde. Jedoch auch das WHG 2010 setzt eine solche Frist (nämlich bis 2013) (§ 76 II WHG 2010). Auch hier sind im deutschen Recht keine Konsequenzen bei Nichterfüllung festgesetzt. Das WHG 2010 schaffte die 2005 eingeführten Hochwasserschutzpläne und auch die Kategorie der „überschwemmungsgefährdeten Bereiche“ wieder ab. Diese Kategorie stand ohnehin in der Kritik, ein zu schwa-

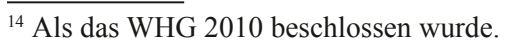


ches Instrument zu sein, unfähig den Flüssen mehr Raum zu geben (Reinhardt 2008, S. 356; Rolfsen 2009, S. 770). Das WHG 2010 führte jedoch erstmals ein Bauverbot für Überschwemmungsgebiete ein, was 2005 versäumt wurde (Kotulla 2006, S. 135). Obgleich unter bestimmten Voraussetzungen einzelne Bauvorhaben zugelassen werden können, wird diese Regelung als großer Schritt angesehen, um den Flüssen mehr Raum zu geben (Rolfsen 2009, S. 770).

\section{Schlussdiskussion}

Seit den Hochwasserereignissen in den 1990er Jahren ist ein Umdenken im Hochwasserschutz zu beobachten: Den Flüssen soll mehr Raum gegeben werden. Der deutsche Gesetzgeber versucht seitdem Schritt für Schritt, hierfür bessere Regelungen einzuführen (vgl. auch Hartmann 2009, S. 534 ff.). Nach Analyse von Recht und Praxis in SachsenAnhalt werden folgende Schlussfolgerungen zur Diskussion gestellt:

\subsection{Schwache rechtliche Instrumente zum}

Wiederherstellen von Raum für die Flüsse

Als Resümee kann festgehalten werden, dass das heutzutage bestehende Instrumentarium von Wasserwirtschaft und Raumplanung zwar im Laufe der Reformen eine weitere Verbauung von Flusslandschaften effektiver verhindern kann (insbesondere durch die strengeren Regelungen zu Überschwemmungsgebieten), jedoch fehlen nach wie vor Instrumente, die in der Lage sind, den Flüssen tatsächlich mehr Raum zu geben, also z. B. bisher deichgeschützte Gebiete in Retentionsräume umzuwandeln. Zwar werden im Zuge von Polderbauten oder Deichrückverlegungen durchaus Projekte realisiert, die den Flüssen Raum geben, diese werden jedoch oft gegen Verbauungen anderenorts „gegengerechnet“ (MLU LSA 2010, S. 66). Wenn also das Umweltbundesamt 2003 von ,Sichern und Wiederherstellen von Hochwasserrückhalteflächen" spricht, sind Erfolge bei der Sicherung durchaus erkennbar, jedoch liefert das bestehende Recht keine Antwort darauf, wie den Flüssen großräumig mehr Raum gegeben werden kann. ${ }^{15}$

\subsection{Gesetzesnovellen behindern die Praxis}

Die Untersuchung der Umsetzung der Hochwasserschutzkonzeption des Landes Sachsen-Anhalt aus 2003 mit dem

\footnotetext{
${ }^{15}$ Zwar gibt das bestehende Recht die Möglichkeit Polder und Hochwasserrückhaltebecken durch das Instrument der Planfeststellungsverfahren zu realisieren, doch ist dies stets ein sehr langwieriges und an Einzelvorhaben gekoppeltes Verfahren, das nicht der Idee, den Flüssen mehr Raum zu geben, entspricht - es ist vielmehr Ausdruck des technokratischen Zugangs zum Thema Hochwasserschutz in Deutschland.
}

Zielhorizont 2010 zeigte, dass die bisherigen Gesetzesnovellen keinen Durchbruch in der Praxis, um den Flüssen mehr Raum zu geben, erzielen konnten. Vielmehr hinkt die Praxis der Rhetorik „den Flüssen mehr Raum geben“, die in den Gesetzesnovellen häufig benutzt wird, hinterher. Auf Ausführungsebene (also in den Planungsstellen und auf den Sachbearbeiterebenen) können hierfür folgende Gründe identifiziert werden: Erstens behindern die strengeren Regulierungen (z. B. zu Überschwemmungsgebieten) Abstimmungs- und Verhandlungssituationen in der Praxis; zweitens bewirken die zahlreichen Gesetzesnovellen durch permanente Umstrukturierungen und Anpassungen der Verwaltung, dass die tatsächliche Arbeit auf der Strecke bleibt. Dabei können die Hochwasserereignisse 1993 und 1995 am Rhein sowie das Hochwasser 2002 an der Elbe ungeachtet der großen Schäden, die sie verursachten, durchaus als Meilensteine gesehen werden, die die Entwicklung des Wasserrechts vorangetrieben haben. Auf zynische Weise bleibt daher abzuwarten, welche Gesetzesnovellen das nächste Hochwasserereignis nach sich ziehen wird. ${ }^{16}$

\subsection{Dilemma von Recht und Praxis}

Die beiden Schlussfolgerungen bilden ein Dilemma: Auf der einen Seite sind die rechtlichen Instrumente, um den Flüssen Raum zu geben, zu schwach, auf der anderen Seite scheinen strengere rechtliche Regelungen nicht zwingend zu einer effektiveren Umsetzung zu führen. Im Hinblick auf die Umsetzung der EU-Hochwasserrichtlinie ist eine Lösung dieses Problems dringend erforderlich: Im Zuge dieser nun auch ins Nationalrecht überführten EU-Hochwasserpolitik steht die Wasserwirtschaft vor der Herausforderung, künftig nicht mehr nur die Deiche und den Bereich zwischen den Deichen zu bewirtschaften, sondern sie muss in der gesamten (Flussgebiets-)Fläche planen.

\subsection{Neue Formen von Governance}

Ein verbessertes Instrumentarium führt also nicht zwangsläufig zu einer besseren Umsetzung. Das deutsche Wasserrecht und die deutsche Wasserpolitik scheinen immer noch darauf ausgerichtet zu sein, Hochwasserschutz durch eine Zunahme von Kontrolle - also z. B. eine Verschärfung des wasserrechtlichen Instrumentariums $-\mathrm{zu}$ betreiben. Die Umsetzung von Raum für die Flüsse erfordert jedoch weniger eine kontrollierende als vielmehr eine kooperative und anreizorientierte Steuerungsrationalität, um im Zusammenspiel aller Stakeholder - Bodeneigentümer, Raumordnung,

\footnotetext{
${ }^{16}$ Während der Autor diese Zeilen schreibt, führen zahlreiche Nebenflüsse in Deutschland, aber auch der Rhein, die Elbe, die Oder, die Weser sowie die Maas in den Niederlanden Hochwasser. Australien kämpft gerade mit den Folgen eines Extremhochwassers und in Brasilien verursachte ein Starkregenereignis große Schäden.
} 
Bauleitplanung, Landwirtschaft, Ober- und Unterlieger - einen flächenorientierten Hochwasserschutz integrativ umzusetzen.

Ein Umdenken von einem in die Höhe (der Deiche) orientierten Hochwasserschutz zu einem in die Fläche orientierten Risikomanagement erfordert neue Formen von Governance, da künftig mehr Stakeholder an Planung und Umsetzung von Hochwasserschutz (oder Hochwasserrisikomanagement) beteiligt sein werden. Konkret bedeutet dies, dass das Hochwasserschutzinstrumentarium des WHG 2010, insbesondere der Hochwasserrisikomanagementplan (§ 75 WHG 2010), auch zum Erproben neuer Formen von Governance - jenseits des Kontrollparadigmas der bisherigen Hochwassergesetzgebung - genutzt werden sollte.

Open Access Dieser Artikel unterliegt den Bedingungen der Creative Commons Attribution Noncommercial License. Dadurch sind die nichtkommerzielle Nutzung, Verteilung und Reproduktion erlaubt, sofern der/die Originalautor/en und die Quelle angegeben sind.

\section{Interviewverzeichnis}

Bodeneigentümer in Biederitz (drei Interviews), Bitterfeld (drei Interviews), Dessau-Waldersee (vier Interviews) und Torgau (Sachsen) (vier Interviews) (Juni 2008).

Bürgermeister der Gemeinden Biederitz (November 2004) und Gübs (Mai 2008).

Expertengespräche mit Referenten im Ministerium für Landwirtschaft und Umwelt des Landes Sachsen-Anhalt, Magdeburg (November 2004).

Interview Landesplanung: Mitarbeiter im Ministerium für Bau und Verkehr des Landes Sachsen-Anhalt (November 2004 und Juni 2008).

Interviews Regionalplanung: Regionalplaner Magdeburg (November 2004 und Mai 2008) und Regionalplaner Altmark (November 2004 und Mai 2008).

Interviews Stadtplanung: Stadtplaner der Stadt Magdeburg (November 2004 und Mai 2008) und Stadtplaner und Mitarbeiter im Umweltamt der Stadt Dessau-Roßlau (November 2004 und Juni 2008).

Interview Wasserwirtschaft: Mitarbeiter des Landebetriebes für Hochwasserschutz und Wasserwirtschaft Sachsen-Anhalt, Wittenberg (November 2004 und Mai 2008).

\section{Gesetzestexte}

Baugesetzbuch (BauGB) in der Fassung vom 3. Mai 2005 (mit den Änderungen des Hochwasserschutzgesetzes vom 3. Mai 2005) (BGB1. I 2005, Nr. 26, 1224).

Baugesetzbuch (BauGB) in der Fassung vom 23. September 2004 (BGB1. I 2004, Nr. 52, 2414).

Hochwasserrichtlinie (Richtlinie über die Bewertung und das Management von Hochwasserrisiken) vom 6. November 2007 (Amtsblatt der Europäischen Union 2007 [DE] L288, 27-34).

Hochwasserschutzgesetz (Gesetz zur Verbesserung des vorbeugenden Hochwasserschutzes) vom 3. Mai 2005 (BGB1. I 2005, Nr. 26, 1224).

Landesplanungsgesetz des Landes Sachsen-Anhalt (LP1G LSA) in der Fassung vom 28. April 1998 (GVB1. LSA 1998, Nr. 16, 255).
Raumordnungsgesetz (ROG) vom 18. August 1997 (BGB1. I 1997, Nr. 59, 2081) mit den Änderungen des Europarechtsanpassungsgesetzes Bau vom 24. Juni 2004 (BGB1. I 2004, Nr. 31, 1359).

Raumordnungsgesetz (ROG) in der Fassung vom 3. Mai 2005 (mit den Änderungen des Hochwasserschutzgesetzes vom 3. Mai 2005), (BGB1. I 2005, Nr. 26, 1224).

Wassergesetz für das Land Sachsen-Anhalt (WG LSA) in der Fassung vom 21. April 1998, (GVB1. LSA 1998, Nr. 15, 186).

Wassergesetz für das Land Sachsen-Anhalt (WG LSA) in der Fassung vom 12. April 2006, (GVBl. LSA 2006, Nr. 15, 248).

Wasserhaushaltsgesetz (Gesetz zur Ordnung des Wasserhaushalts 1996) (WHG) vom 12. November 1996 (BGB1. I 1996, Nr. 58, 1695).

Wasserhaushaltsgesetz (Gesetz zur Ordnung des Wasserhaushalts 2005) (WHG) vom 3. Mai 2005 (mit den Änderungen des Hochwasserschutzgesetzes vom 3. Mai 2005) (BGB1. I, Nr. 26, 1224).

Wasserhaushaltsgesetz (Gesetz zur Ordnung des Wasserhaushalts 2010) (WHG) vom 31. Juli 2009 (BGB1. I 2009, Nr. 51, 2585).

Zweites Gesetz zur Erleichterung von Investitionen im Land SachsenAnhalt (Zweites Investitionserleichterungsgesetz LSA) in der Fassung vom 16. Juli 2003 (GVBI. LSA 2003, Nr. 26, 157).

\section{Literatur}

Breuer, R. (2006): Die neuen wasserrechtlichen Instrumente des Hochwasserschutzgesetzes vom 03.05.2005. In: Natur und Recht 28, $10,614-623$

Corell, C. (1996): Schaffung und Bewahrung von Retentionsräumen zum Zwecke des Hochwasserschutzes. In: Umwelt- und Planungsrecht 16, 7, 246-253.

Cormann, P. (2008): Kommentar zum WHG. In: Giesberts, L.; Reinhardt, M (Hrsg.): Umweltrecht. München.

Czychowski, M. (1998): Wasserhaushaltsgesetz - Kommentar. München.

BMVBW (Bundesministerium für Verkehr-, Bau- und Wohnungswesen) (2003): Flusskonferenz 2002 - Gemeinsame Konferenz zum vorbeugenden Hochwasserschutz. Berlin.

Bundesregierung (2005): Bericht der Bundesregierung über die nach der Flusskonferenz vom 15. September 2002 eingeleiteten Maßnahmen zur Verbesserung des vorbeugenden Hochwasserschutzes. Berlin.

Deutscher Bundestag (2009a): Drucksache 16/12786: Entwurf eines Gesetzes zur Neuregelung des Wasserrechts vom 27. April 2009. Berlin.

Deutscher Bundestag (2009b): Drucksache 16/13491: Änderungsantrag zum Entwurf eines Gesetzes zur Neuregelung des Wasserrechts vom 18. Juni 2009. Berlin.

Europäische Union (2007): Richtlinie 2007/60/EG. In: Amtsblatt der Europäischen Union L 288, 27-34.

Greiving, S. (2002): Räumliche Planung und Risiko. München.

Grünewald, U. (2005): Vom Hochwasser-,,Schutzversprechen“ zum Hochwasser-,,Risikomanagement“. In: Jüpner, R. (Hrsg.): Hochwassermanagement. Aachen, 5-22. = Magdeburger Wasserwirtschaftliche Hefte, Band 1/2005.

Hartmann, T. (2005): Hochwasserschutz durch räumliche Planung - Handlungsempfehlungen für Abstimmungsprozesse zwischen den Akteuren in Sachsen-Anhalt. Unveröffentlichte Diplomarbeit an der Fakultät Raumplanung der Universität Dortmund.

Hartmann, T. (2009): Clumsy Floodplains and the Law - Towards a Responsive Land Policy for Extreme Floods. In: Built Environment 35, 4, 531-544.

Hartmann, T. (2010): Reframing Polyrational Floodplains - Land Policy for Large Areas for Temporary Emergency Retention. In: Nature \& Culture 5, 1, 15-30. 
Hartmann, T. (2011): Clumsy Floodplains - Responsive Land Policy for Extreme Floods. Farnham, Surrey.

Haupter, B.; Heiland, P. (2002): Vorsorgender Hochwasserschutz: Verantwortung der Raumordnung? In: RaumPlanung 104, 233-235.

Heiland, P. (2002): Vorsorgender Hochwasserschutz durch Raumordnung, interregionale Kooperation und ökonomischen Lastenausgleich. Darmstadt. = Schriftenreihe des Instituts WAR, Band 143.

Jacobitz, K. (2005): Wasserwirtschaft. In: Akademie für Raumforschung und Landesplanung (ARL) (Hrsg.): Handwörterbuch der Raumordnung. Hannover, 1087-1093.

Johnson, C. L.; Priest, S. J. (2008): Flood risk management in England. In: Water Resources Development 24, 4, 513-526.

Knopp, G. (2010): Das neue Wasserhaushaltsrecht. München.

Kotulla, M. (2006): Das Gesetz zur Verbesserung des vorbeugenden Hochwasserschutzes. In: Neue Zeitschrift für Verwaltungsrecht $25,2,129-135$

Landtag Sachsen-Anhalt (2003): Drucksache 4/610: Entwurf eines Zweiten Investitionserleichterungsgesetzes vom 5. März 2003. Magdeburg.

Landtag Sachsen-Anhalt (2004): Drucksache 4/1789: Entwurf eines Vierten Gesetzes zur Änderung des Wassergesetzes für das Land Sachsen-Anhalt vom 2. September 2004. Magdeburg.

LAWA (Länderarbeitsgemeinschaft Wasser) (1995): Leitlinien für einen zukunftsweisenden Hochwasserschutz. Berlin.

MKRO (Ministerkonferenz für Raumordnung) (1995): Beiträge räumlicher Planungen zum vorbeugenden Hochwasserschutz. Beschluss der Ministerkonferenz für Raumordnung vom 8. März 1995. Berlin.

MKRO (Ministerkonferenz für Raumordnung) (1996): Grundsätze und Ziele der Raumordnung und Landesplanung zu einem grenzübergreifenden vorbeugenden Hochwasserschutz an Fließgewässern. Beschluss der Ministerkonferenz für Raumordnung vom 29. März 1996. Berlin.

MKRO (Ministerkonferenz für Raumordnung) (1998): Raumordnung und vorbeugender Hochwasserschutz. Beschluss der Ministerkonferenz für Raumordnung vom 4. Juni 1998. Berlin.

MKRO (Ministerkonferenz für Raumordnung) (2000): Handlungsempfehlungen der Ministerkonferenz für Raumordnung zum vorbeugenden Hochwasserschutz. Empfehlungen der MKRO vom 14. Juni 2000. Berlin.

MLU LSA (Ministerium für Landwirtschaft und Umwelt SachsenAnhalt) (2003): Hochwasserschutzkonzeption des Landes Sachsen-Anhalt bis 2010. Magdeburg.
MLU LSA (Ministerium für Landwirtschaft und Umwelt SachsenAnhalt) (2010): Hochwasserschutzkonzeption des Landes Sachsen-Anhalt bis 2020. Magdeburg.

Monstadt, J.; Moss, T. (2008): Policy innovations in the aftermath of a disaster: contexts of floodplain restoration in Germany. In: Moss, T.; Monstadt, J (Hrsg.): Restoring the floodplains in Europe. Policy contexts and project experiences. London, 63-87.

Pape, K. A. (2010): Wasserhaushaltsrecht nach der Novelle des Wasserhaushaltsgesetzes. In: Landmann, R.; Rohmer, G (Hrsg.): Umweltrecht Kommentar. Band III. München.

Petrow, T.; Thieke, A. H.; Kreibich, H.; Bahlburg, C. H.; Merz, B. (2006): Improvements on flood alleviation in Germany. In: Environmental Management 38, 5, 717-732.

Prittwitz, V. von (1994): Politikanalyse. Opladen.

Reinhardt, M. (2008): Identität und Zukunft des Wasserrechts als Bestandteil eines Umweltgesetzbuchs. In: Zeitschrift für Umweltrecht 7-8, 352-357.

Rolfsen, M. (2009): Das neue Wasserhaushaltsgesetz. In: Natur und Recht 31, 765-771.

Roth, D.; Warner, J. (2007): Flood risk uncertainty and changing river protection policy in The Netherlands: the case of ,calamity polders'. In: Tijdschrift voor Economische en Sociale Geografie 98, 4, 519-525.

Sächsisches Staatsministerium des Inneren (2006): ELLA - Vorsorgende Hochwasserschutzmaßnahmen durch transnationale Raumordnung für das Einzugsgebiet der Elbe. Dresden.

Schneider, S. (2005): Rechtliche Instrumente des Hochwasserschutzes in Deutschland. Berlin. = Wasserrecht und Wasserwirtschaft, Band 40.

Strobl, T.; Zunic, F. (2006): Wasserbau. Aktuelle Grundlagen - neue Entwicklungen. Berlin, Heidelberg.

Umweltbundesamt (1998): Ursachen der Hochwasserentstehung und ihre anthropogene Beeinflussung. Berlin. $=$ UBA Texte 18/98

Umweltbundesamt (1999): Anforderungen des vorsorgenden Hochwasserschutzes an Raumordnung, Landes-/Regionalplanung, Stadtplanung und die Umweltfachplanungen. Berlin. = UBA Texte 45/99.

Umweltbundesamt (2003): Sichern und Wiederherstellen von Hochwasserrückhalteflächen. Berlin. = UBA Texte 34/03. 